\title{
DISSEMINATED HISTOPLASMOSIS
}

\author{
MD. SHAHRIAR MAHBUB,${ }^{1}$ HAM NAZMUL AHASAN,${ }^{2}$ MD TITU MIAH,${ }^{3}$ MD BILLAL ALAM, ${ }^{4}$ RATAN \\ DAS GUPTA, ${ }^{3}$ KHAN MOHAMMAD ARIF, ${ }^{5}$ MUNTASIR HASNAIN ${ }^{6}$
}

\begin{abstract}
A case of disseminated histoplasmosis in a 56-year-old apparently healthy male without any history of travel to endemic zone is described. The patient presented with fever with cough, respiratory distress and disorientation. Physical findings include fever, shortness of breath, reduced level of consciousness and hypotension. Diagnosis was confirmed by presence of Histoplasma capsulatum in bone marrow aspirate. This report illustrates the importance of recognizing the possibility of histoplasmosis in Bangladesh where mimickers of histoplasmosis like pulmonary tuberculosis and visceral leishmaniasis are extremely common.
\end{abstract}

Keywords: Histoplasmosis, endemic mycoses, disseminated histoplasmosis, Histoplasma capsulatum, Bangladesh

\section{Introduction}

Histoplasmosis is the most common endemic mycosis in human. Histoplasma capsulatum is a dimorphic fungus distributed worldwide, but endemic in the Americas, Africa, and Asia. ${ }^{1}$ It was first described by Samuel Darling in 1906 in an adult patient who presumably died of military tuberculosis. ${ }^{2}$ Initial infection is through respiratory tract through which it enters the reticuloendothelial system and resides in macrophages. Most individuals with intact cellular immunity are asymptomatic or have mild pulmonary symptoms. Severe disseminated histoplasmosis develops in people with primary or secondary deficiency of cellular immunity. Immunosuppressed conditions due either to infections like AIDS or drugs raise the possibility of histoplasmosis. Approximately, 10\% cases of histoplasmosis develop into progressive disseminated histoplasmosis (PDH). ${ }^{3}$ Disseminated histoplasmosis may present as acute PDH with fever, malaise, cough mimicking pulmonary tuberculosis. Chronic PDH manifests as fever, sweats, weight loss, organomegaly, lymphadenopathy.

In Bangladeshi context, reporting of histoplasmosis in medical literature is very rare. We present a case of histoplasmosis in an apparently healthy individual who initially had pulmonary involvement and later progressed to develop disseminated histoplasmosis.

\section{Case Report}

A 56 years old previously healthy male was admitted with the complaints of fever with dry cough of 11 months duration, respiratory distress for 7 days and disorientation for 2 days. His previous medical history was unremarkable except for the fact that he was given anti-tuberculous drugs for a presumed diagnosis of pulmonary tuberculosis for 5 months before admission without any improvement. The patient denied any history of travel or sexual exposure. He was not on any drug that might have induced immunosuppression.

On general examination, the patient was febrile with a recorded temperature of $100^{\circ} \mathrm{F}\left(37.8^{\circ} \mathrm{C}\right)$, level of consciousness on Glasgow Coma Scale 10, tachypnic, hypotensive (blood pressure $80 / 60 \mathrm{~mm} \mathrm{Hg}$ ). Other parameters were normal. Systemic examination revealed no abnormality. The patient's laboratory investigation results showed anemia with hemoglobin $9 \mathrm{~g} / \mathrm{dL}$, ESR $60 \mathrm{~mm}$ in $1^{\text {st }}$ hour, total WBC count $9000 /$ $\mathrm{cm}^{3}$, normal platelet count, serum creatinine $2.3 \mathrm{mg} /$ dL. Sputum for Acid-fast bacillus was negative. Mantoux test was negative. HIV screening returned

1. Postgraduate trainee, Department of Medicine, Dhaka Medical College Hospital, Dhaka

2. Professor, Department of Medicine, Dhaka Medical College, Dhaka

3. Assistant Professor, Department of Medicine, Dhaka Medical College, Dhaka

4. Associate Professor, Department of Medicine, Dhaka Medical College, Dhaka

5. Indoor Medical Officer, Department of Medicine, Dhaka Medical College Hospital

6. Assistant Registrar, Department of Medicine, Dhaka Medical College Hospital

Correspondence: Dr. Md. Shahriar Mahbub, Postgraduate trainee, Department of Medicine, Dhaka Medical College, Dhaka, E-mail: shahriar04@yahoo.com 
negative (Figure 1). Cerebrospinal fluid study was normal. Chest X-ray showed bilateral diffuse infiltrate. Peripheral blood smear was normal. Bone marrow aspirate showed plenty of intracellular

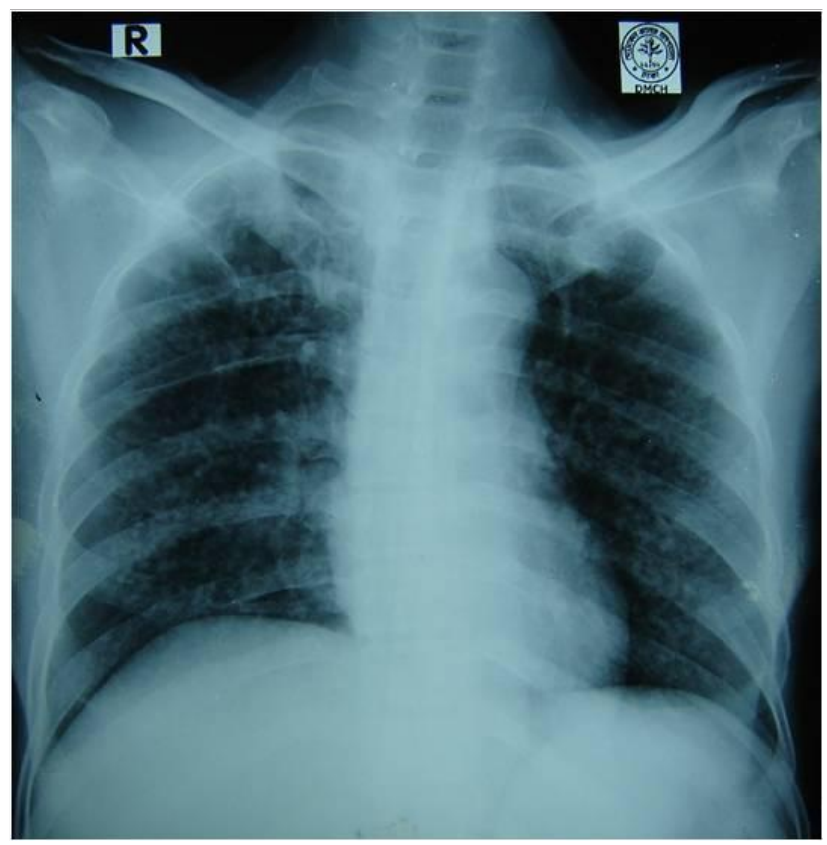

Fig.-1: Chest X-ray showing bilateral diffuse pulmonary infiltrate

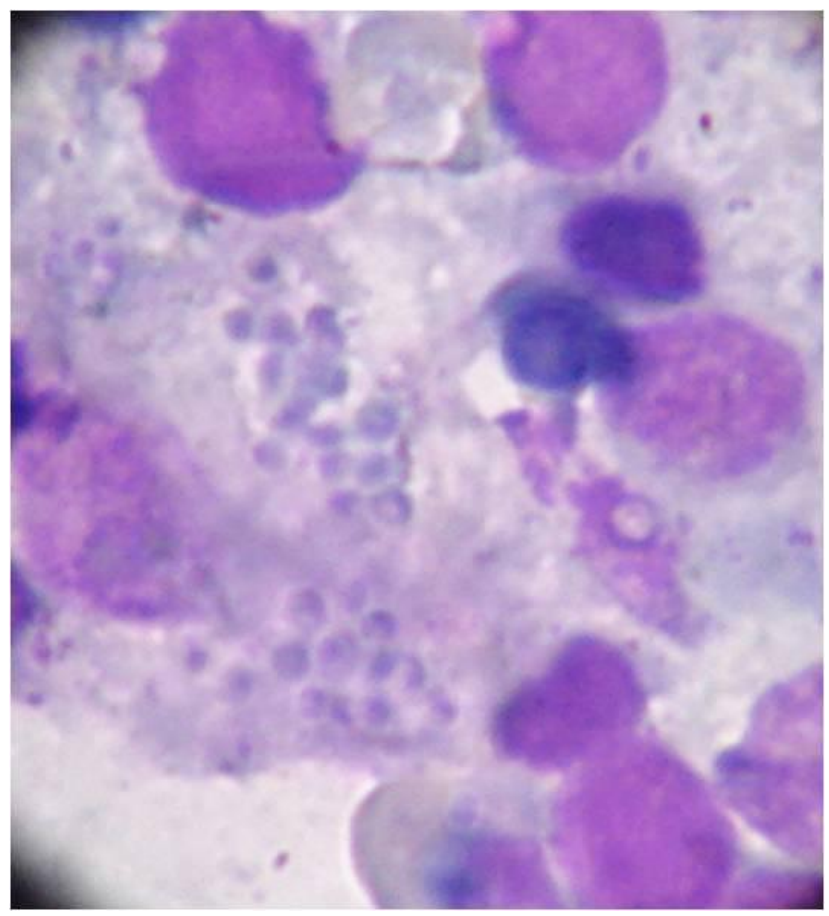

Fig.-2: Bone marrow aspirate showing numerous Histoplasma Capsulatum (Leishman stain) rounded to oval parasites with eccentric crescent shaped nucleus suggestive of Histoplasma Capsulatum (Figure 2).

The patient was started on intravenous amphotericin B. His condition improved initially with temperature returning to baseline. Unfortunately, the patient developed aspiration pneumonia on $5^{\text {th }}$ post admission day and expired.

\section{Discussion}

Histoplasmosis is endemic in parts of North and South America, Asia and Africa. It is the most common endemic mycoses in the United States. The causative agent $H$. capsulatum grows in soil laden with excreta of chickens, pigeons, starlings, blackbirds, and bats. ${ }^{4}$ Exposure occurs through contact with chicken houses, soil containing large amount of bird or bat guano and old houses or caves known to be bat roosts. ${ }^{4}$

Incidence of histoplasmosis in non-endemic areas is far fewer than endemic areas. Commonly, histoplasmosis occurs in non-endemic areas due to travel or residence in endemic areas. Additionally, it may be found outside endemic areas where microfoci of Histoplasma are the source of infection. ${ }^{5}$ Incidence figures of histoplasmosis in Bangladesh are unavailable. Sporadic cases have been reported in different literature. A review of literature revealed only 3 cases of histoplasmosis from Bangladesh including one patient who had a renal transplant and was on immunosuppressive agents. ${ }^{2,4,6}$

The severity of clinical manifestation depends on size of the inoculum, underlying health of the patient and immune status to Histoplasma. ${ }^{1}$ Majority of patients with AIDS present with disseminated infection while immunocompetent individuals demonstrate clinical features ranging from asymptomatic infection to rapidly fatal pulmonary infection. In between, patients may develop a variety of clinical manifestations including acute or subacute pulmonary disease, progressive disseminated disease, pericarditis, arthritis or, less commonly, fibrosing mediastinitis. $^{1}$

Disseminated histoplasmosis may present either as self-limited disease or progressive disseminated histoplasmosis. ${ }^{5}$ The self-limited condition is found in acute histoplasmosis. It occurs during the first few weeks of illness when hematogenous dissemination leads to development of various 
clinical features. Hepatomegaly, splenomegaly, bone marrow suppression, elevated hepatic enzymes are some of the features. Calcified granuloma in the spleen is a common finding in people living in the endemic zone. Blood culture is rarely positive in acute stage. Specific cell-mediated immunity plays an important role in controlling the infection in lung and extrapulmonary tissues.

Progressively disseminated histoplasmosis usually occurs either in patients at extremes of age or in patients with an underlying immune deficiency state due to AIDS, leukemia, lymphoma, systemic lupus erythematosus, systemic corticosteroids, solid organ transplantation, anti-tumor necrosis factor agents. Not all patients harbor an immunodeficient status. ${ }^{5}$ Unidentified mechanism leading to immune deficiency is thought to be behind these cases. Ongoing research has identified defects in interferon$\alpha /$ interleukin-12 pathway as a possible explanation in otherwise healthy individuals who develop progressive disseminated histoplasmosis. ${ }^{1}$ The clinical features include fever, weight loss, fatigue, respiratory complaints like cough and shortness of breath. Hepatomegaly, splenomegaly, lymphadenopathy, bone marrow involvement are found in less than $50 \%$ of cases. Less common manifestations include oropharyngeal ulcers, gastrointestinal hemorrhage, adrenal insufficiency, subacute to chronic meningitis and endocarditis. Few patients may present with acute shock-like episodes with hypotension and coagulopathy. In significant number of cases, the only findings are fever and progressive weight loss. ${ }^{5}$

Laboratory diagnosis of histoplasmosis can be made by growth of histoplasma in culture. Bone marrow aspirate, peripheral blood smear, lymph node biopsy, bronchoalveolar lavage fluid, tranbrochial biopsy specimen and biopsy from cutaneous lesions can be used for diagnosis. ${ }^{4}$ Among all these, bone marrow examination has the highest diagnostic yield. ${ }^{7}$ Other laboratory abnormalities include anemia, leukopenia, pancytopenia, elevated liver enzymes, increased ferritin and serum lactate dehydrogenase. Antigen detection in urine and serum by radioimmunoassay is highly sensitive in disseminated infection. It can also be used for monitoring response to treatment especially in AIDS patient. The most common finding on chest imaging is diffuse interstitial or reticulonodular infiltrates and military infiltrates. ${ }^{5}$ Chest X-ray in chronic pulmonary histolpasmosis may also show upper lobe involvement with cavitation leading to a misdiagnosis of pulmonary tuberculosis.

The recommended treatment regimen for disseminated histoplasmosis is liposomal Amphotericin B for 1-2 weeks followed by oral itraconazole for at least 12 months. ${ }^{8}$ Suppressive therapy with itraconazole may be required in immunocompromised patients like AIDS or organ transplant patient. ${ }^{7}$ Liposomal Amphotericin B is better than conventional Amphotericin B in terms of toxicity and therapeutic efficacy.

Our patient presented with chronic respiratory complaints of cough with fever followed by respiratory distress and disorientation. Before admission, his initial symptoms and imaging findings led to the diagnosis of pulmonary tuberculosis which is widely prevalent in Bangladesh. Indeed, histoplasmosis is under-reported from Bangladesh due to low index of suspicion and lack of diagnostic facility. Another possible explanation of underdiagnosis is that disseminated histoplasmosis resembles visceral leishmaniasis in many aspects with features of fever, weight loss, hepatosplenomegaly. Moreover, both of these are responsive to Amphotericin B. Our patient did not have organomegaly which is found in only one-third of patients. Bone marrow involvement is suggested by anemia and raised ESR. These findings along with thrombocytopenia are the most common hematological abnormalities.

Literature review revealed only 3 cases of histoplasmosis from Bangladesh - a 69-year-old male with oral ulcer, hepatosplenomegaly, anemia and leucopenia. ${ }^{6}$ Another patient was diagnosed in a neighboring country and the third patient, a renal transplant receiver on immunosuppressive therapy, was diagnosed and treated in the United States (US) where he returned from Bangladesh after a visit. This patient did not travel to or was living in histoplasmosis endemic zone of US, namely Ohio and Mississippi River Valleys. The authors concluded that the temporal relationship of his visit to Bangladesh and onset of symptoms suggested that he was infected after he came in contact with chicken houses in Bangladesh. ${ }^{4}$ Reports from India suggest 
the presence of endemic focus in abandoned houses containing bat guano and in forest soil. ${ }^{9,10}$

In conclusion, our case illustrates the importance of high index of suspicion for diagnosing histoplasmosis in a patient presenting with features suggestive of pulmonary tuberculosis but failing to respond to antiTB after a reasonable period of time. Increasing prevalence of AIDS, organ transplantation and expanding international travel is likely to heighten the possibility of histoplasmosis in Bangladesh. Physicians need to alert themselves to this underdiagnosed infectious disease which is ultimately fatal if left untreated.

\section{Acknowledgement}

We would like to thank Dr. Salma Afrose, Associate Professor, Department of Hematology, Dhaka Medical College for her invaluable support with bone marrow study.

\section{References}

1. Wheat LJ, Conger NG. Histoplasmosis. In: Hospenthal DR, Rinaldi MG, eds. Diagnosis and Treatment of Human Mycoses. New Jersey: Humana Press, 2008:318-329.

2. Subramanian S, Abraham OC, Rupali P, et al. Disseminated Histoplasmosis. J Assoc Physicians India 2005;53:185-189.
3. Joshi SA, Kagal AS, Bharadwaj RS, et al. Disseminated Histoplasmosis. Indian J Med Microbiol 2006;24:297-298.

4. Rappo U, Beitler UR, Faulhaber JR, et al. Expanding the horizons of histoplasmosis: disseminated histoplasmosis in a renal transplant patient after a trip to Bangladesh. Transpl Infect Dis 2009;:1-6.

5. Wheat LJ. Histoplasmosis: a review for clinicians from non-endemic areas. Mycoses 2006;49:274-282.

6. Islam N, Chowdhury NA. Histoplasmosis from Bangladesh: a case report. Bangladesh Med Res Counc Bull 1982; 8: 21-24.

7. Doughan A. Disseminated histoplasmosis: Case report and brief review. Travel Med Infect Dis 2006; 4:332-335.

8. Wheat LJ, Freifeld AG, Kleiman MB, et al. Clinical practice guidelines for the management of patients with histoplasmosis: 2007 update by the Infectious Diseases Society of America. Clin Infect Dis 2007; 45:807-825.

9. Randhawa HS, Khan ZU. Histoplasmosis in India: current status. Indian J Chest Dis Allied Sci 1994; 36: 193-213.

10. Goswami RP, Pramanik N, BanerjeeD, et al. Histoplasmosis in eastern India: the tip of the iceberg? Trans R Soc Trop Med Hyg 1999; 93: 540542 . 